\title{
Deformations of smooth functions on 2-torus
}

\author{
Bohdan Feshchenko
}

\begin{abstract}
Let $f$ be a Morse function on a smooth compact surface $M$ and $\mathcal{S}^{\prime}(f)$ be the group of $f$-preserving diffeomorphisms of $M$ which are isotopic to the identity map. Let also $G(f)$ be the group of automorphisms of the Kronrod-Reeb graph of $f$ induced by elements from $\mathcal{S}^{\prime}(f)$, and $\Delta^{\prime}(f)$ be the subgroup of $\mathcal{S}^{\prime}(f)$ consisting of diffeomorphisms which trivially act on the Kronrod-Reeb graph of $f$. The group $\pi_{0} \mathcal{S}^{\prime}(f)$ can be viewed as an analogue of a mapping class group for $f$-preserved diffeomorphisms of $M$. The groups $\pi_{0} \Delta^{\prime}(f)$ and $G(f)$ encode "combinatorially trivial" and "combinatorially nontrivial" counterparts of $\pi_{0} \mathcal{S}^{\prime}(f)$ respectively. In the paper we compute groups $\pi_{0} \mathcal{S}^{\prime}(f), G(f)$, and $\pi_{0} \Delta^{\prime}(f)$ for Morse functions on 2-torus $T^{2}$.
\end{abstract}

Анотація. Гомотопійні властивості функцій Морса на поверхнях вивчались багатьма математиками. Зокрема, компоненти зв'язності простору функцій Морса були описані Х. Цишангом у неопублікованій праці, С. Матвеєвим у роботі О. Кудрявцевої [6] і В. Шарком [20]. Групи кобордизмів простору функцій Морса були описані К. Ікегамі і О. Саекі [4], а також Б. Кальмаром [5]. Інший підхід до вивчення деформацій функцій Морса за допомогою дослідження стабілізаторів і орбіт цих функцій був запропонований С. Максименком $[10,11,12,14,15]$ і О. Кудрявцевою $[7,8]$.

Група дифеоморфізмів $\mathcal{D}(M)$ діє справа на просторі гладких функцій $C^{\infty}(M)$ за таким правилом:

$$
C^{\infty}(M) \times \mathcal{D}(M) \rightarrow C^{\infty}(M), \quad(f, h) \mapsto f \circ h .
$$

Для функції $f \in C^{\infty}(M)$ нехай $\mathcal{S}(f)=\{h \in \mathcal{D}(M) \mid f \circ h=f\}$ - стабілізатор $f$ і $\mathcal{O}(f)=\{f \circ h \mid h \in \mathcal{D}(M)\}$ - орбіта $f$ відносно цієї дії. Наділимо простори $\mathcal{D}(M)$ і $C^{\infty}(M)$ сильними топологіями Уїтні. Ці топології індукують деякі топології на просторах $\mathcal{S}(f)$ і $\mathcal{O}(f)$. Нехай також $\mathcal{D}_{\mathrm{id}}(M)$ та $\mathcal{S}_{\text {id }}(f)$ - зв'язні компоненти тотожнього відображення, а $\mathcal{O}_{f}(f)$ - зв'язна компонента $\mathcal{O}(f)$, що містить $f$. Покладемо $\mathcal{S}^{\prime}(f)=\mathcal{S}(f) \cap \mathcal{D}_{\text {id }}(M)$.

Нехай $\Gamma_{f}$ - граф функції $f$. Тоді будь-який $h \in \mathcal{S}^{\prime}(f)$ індукує гомеоморфізм графу $\Gamma_{f}$. Групу таких гомеоморфізмів позначимо через $G(f)$. Нехай $\Delta^{\prime}(f)$ - підгрупа в $\mathcal{S}^{\prime}(f)$, що складається із гомеоморфізмів, що

Keywords: surface, isotopy, Morse function, wreath product 
тривіально діють на графі функції $f$, а також є ізотопними тотожньому відображенню. С. Максименко досліджував групи $\pi_{0} \Delta^{\prime}(f)$, а також фактор-групу $G(f)=\pi_{0} \mathcal{S}^{\prime}(f) / \pi_{0} \Delta^{\prime}(f)$.

Група $\pi_{0} \mathcal{S}^{\prime}(f)$ є певним аналогом групи класів відображень для дифеоморфізмів, що зберігають функцію. При такій аналогії групи $\pi_{0} \Delta^{\prime}(f)$ і $G(f)$ відображають "комбінаторно тривіальну" і "комбінаторно нетривіальну" частини $\mathcal{S}^{\prime}(f)$.

У серії робіт $[17,9,16,2]$ досліджувалась алгебраїчна структура фундаментальної групи $\mathcal{O}_{f}(f)$ для функцій Морса. Було показано, що вона може бути обчислена за допомогою гомотопійної інформації про обмеження заданої функції $f$ на підповерхні 2-тора, що є дисками та циліндрами узгодженими з $f$. Дана робота присвячена алгебраїчному опису груп $\pi_{0} \mathcal{S}^{\prime}(f), \pi_{0} \Delta^{\prime}(f)$ і $G(f)$ для функцій Морса на 2-торі.

\section{INTRODUCTION}

Homotopy properties of Morse functions on surfaces were studied by many authors. E.g. connected components of the space of Morse functions were computed in the unpublished paper by H. Zieschang, by S. Matveev in the paper by E. Kudryavtseva [6], and V. Sharko [20], cobordism groups of the space of Morse functions on surfaces were described by K. Ikegami and O. Saeki [4], and B. Kalmar [5]. Homotopy groups of stabilizers and orbits of Morse functions on surfaces with respect to the action of diffeomorphism groups were studied by S. Maksymenko [10, 11, 12, 14, 15] and E. Kudryavtseva [7, 8]. We will give an overview of these results.

Let $M$ be a smooth compact surface and $X$ be a closed (possible empty) subset of $M$. The group $\mathcal{D}(M, X)$ of diffeomorphisms fixed on some neighborhood of $X$ acts on the space of smooth functions $C^{\infty}(M)$ by the rule: $C^{\infty}(M) \times \mathcal{D}(M, X) \rightarrow C^{\infty}(M),(f, h) \mapsto f \circ h$. With respect to this action we denote by

$$
\begin{gathered}
\mathcal{S}(f, X)=\{h \in \mathcal{D}(M, X) \mid f \circ h=f\}, \\
\mathcal{O}(f, X)=\{f \circ h \mid h \in \mathcal{D}(M, X)\}
\end{gathered}
$$

the stabilizer and the orbit of $f \in C^{\infty}(M)$ respectively. Endow strong Whitney $C^{\infty}$-topologies on $C^{\infty}(M)$ and $\mathcal{D}(M, X)$. Then for each $f \in C^{\infty}(M)$ these topologies induce some topologies on $\mathcal{S}(f, X)$ and $\mathcal{O}(f, X)$. We denote by $\mathcal{D}_{\text {id }}(M, X), \mathcal{S}_{\text {id }}(f, X)$, and $\mathcal{O}_{f}(f, X)$ connected components of the identity $\operatorname{map} \operatorname{id}_{M}$ of $\mathcal{D}(M, X), \mathcal{S}(f, X)$, and the component of $\mathcal{O}(f, X)$ containing $f$ respectively. If $X=\varnothing$ we will omit the symbol " $\varnothing$ " from our notations, i.e., set $\mathcal{D}(M):=\mathcal{D}(M, \varnothing), \mathcal{S}(f):=\mathcal{S}(f, \varnothing), \mathcal{O}(f):=\mathcal{O}(f, \varnothing)$, and so on.

By a Morse function $f$ on a $M$ we will mean a smooth function which satisfies the following conditions: 
- all critical points of $f$ are non-degenerate and belong to the interior of $M$;

- the function $f$ takes constant values on each boundary component of $M$.

Notice that if $N \subset M$ is a subsurface whose boundary components are regular components of some level-sets of a Morse function $f: M \rightarrow \mathbb{R}$, then the restriction $\left.f\right|_{N}$ is a Morse function as well in the sense of the above definition.

Theorem 1.1. [19, 10, 14, 13]. Let $f$ be a Morse function on a smooth compact surface $M$, and $X$ be a closed (possibly empty) subset of $M$ consisting of finitely many connected components of some level-sets of $f$ and some critical points of $f$. Then the following statements hold.

(1) The map $p: \mathcal{D}_{\mathrm{id}}(M, X) \rightarrow \mathcal{O}(f, X)$ defined by $p(h)=f \circ h$ is a Serre fibration with the fiber $\mathcal{S}(f, X)$. Hence $p\left(\mathcal{D}_{\mathrm{id}}(M)\right)=\mathcal{O}_{f}(f)$, and the restriction $\left.p\right|_{\mathcal{D}_{\mathrm{id}}(M)}: \mathcal{D}_{\mathrm{id}}(M) \rightarrow \mathcal{O}_{f}(f, X)$ is also a Serre fibration with the fiber $\mathcal{S}^{\prime}(f, X)=\mathcal{S}(f) \cap \mathcal{D}_{\text {id }}(M, X)$.

(2) $\mathcal{O}_{f}(f, X)=\mathcal{O}_{f}(f, X \cup \partial M)$, so $\pi_{k}\left(\mathcal{O}_{f}(f, X)\right)=\pi_{k}\left(\mathcal{O}_{f}(f, X \cup \partial M)\right)$ for $k \geqslant 1$.

(3) Suppose that either $f$ has a saddle point or $M$ is a non-orientable surface. Then $\mathcal{S}_{\mathrm{id}}(f)$ is contractible, $\pi_{k} \mathcal{O}_{f}(f)=\pi_{k} M, k \geqslant 3, \pi_{2} \mathcal{O}_{f}(f)=0$, and for $\pi_{1} \mathcal{O}_{f}(f)$ we have the following short exact sequence of groups:

$$
\pi_{1} \mathcal{D}_{\mathrm{id}}(M) \stackrel{p_{1}}{\longrightarrow} \pi_{1} \mathcal{O}_{f}(f) \stackrel{\partial_{1}}{\longrightarrow} \pi_{0} \mathcal{S}^{\prime}(f)^{1}
$$

Moreover the group $p_{1}\left(\pi_{1} \mathcal{D}_{\mathrm{id}}(M)\right)$ is contained in the center of $\pi_{1} \mathcal{O}_{f}(f)$.

(4) If $\chi(M)<|X|$, then $\mathcal{D}_{\mathrm{id}}(M, X)$ is contractible, $\pi_{k} \mathcal{O}_{f}(f, X)=0$ for $k \geqslant 2$, and the boundary map

$$
\partial_{1}: \pi_{1} \mathcal{O}_{f}(f, X) \longrightarrow \pi_{0} \mathcal{S}^{\prime}(f, X)
$$

is an isomorphism.

We recall the definition of the map $\partial_{1}$. Let $\omega:[0,1] \rightarrow \mathcal{O}_{f}(f), \omega_{0}=\omega_{1}$ be a loop in $\mathcal{O}_{f}(f)$ based in $f$. Since $p$ is a Serre fibration, it follows that there exists an isotopy $h: M \times[0,1] \rightarrow M$ such that $\omega_{t}=f \circ h_{t}, h_{0}=\mathrm{id}$, and $h_{1} \in \mathcal{S}^{\prime}(f)$, i.e.,$h_{1}$ is such that $f \circ h_{1}=f$. Then the map $\partial_{1}$ is defined by the formula $\partial([\omega])=\left[h_{1}\right] \in \pi_{0} \mathcal{S}^{\prime}(f)$.

\footnotetext{
${ }^{1}$ Throughout the text injective and surjective maps of groups will be also denoted by hooked $\hookrightarrow$ and double-headed arrows $\rightarrow$ respectively.
} 


\subsection{Automorphisms of graphs of functions on surfaces. Let}

$$
f: M \rightarrow \mathbb{R}
$$

be a Morse function on a smooth compact oriented surface $M$ and $c$ be a real number. A connected component $C$ of the level-set $f^{-1}(c)$ is called critical, if $C$ contains at most one critical point of $f$, otherwise $C$ is regular. Let $\Xi$ be a partition of $M$ into connected components of level-sets of $f$. It is well known that the quotient-space $\Gamma_{f}=M / \Xi$ has a structure of a 1-dimensional CW complex called the graph of $f$, or Kronrod-Reeb graph of $f$. Let also $p_{f}: M \rightarrow \Gamma_{f}$ be a projection map. Then $f$ can be represented as the composition:

$$
f=\hat{f} \circ p_{f}: M \stackrel{p_{f}}{\longrightarrow} \Gamma_{f} \stackrel{\hat{f}}{\longrightarrow} \mathbb{R} .
$$

Denote by Aut $\left(\Gamma_{f}\right)$ the group of homeomorphisms of the graph $\Gamma_{f}$. Note that each $h \in \mathcal{S}(f, X)$ preserves level-sets of $f$. Hence, $h$ induces the homeomorphism $\rho(h)$ of $\Gamma_{f}$ such that the following diagram

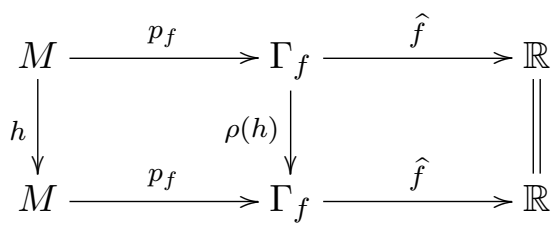

commutes, and the correspondence $h \mapsto \rho(h)$ is a homomorphism

$$
\rho: \mathcal{S}(f, X) \rightarrow \operatorname{Aut}\left(\Gamma_{f}\right) .
$$

One can check that the image $\rho(\mathcal{S}(f, X))$ is a finite subgroup in $\operatorname{Aut}\left(\Gamma_{f}\right)$. The image $\rho\left(\mathcal{S}^{\prime}(f, X)\right)$ in Aut $\left(\Gamma_{f}\right)$ will be denoted by $G(f, X)$.

Let $\Delta(f, X)$ be the normal subgroup of $\mathcal{S}(f, X)$ consisting of diffeomorphisms which leave invariant every connected component of each level set of $f$, and $\Delta^{\prime}(f, X)$ be the following intersection $\Delta(f, X) \cap \mathcal{D}_{\text {id }}(M, X)$. It is known that $\pi_{0} \Delta^{\prime}(f, X)$ is a free abelian group and $\operatorname{Ker} \rho=\pi_{0} \Delta^{\prime}(f, X)$. So the following sequence of groups is exact

$$
\pi_{0} \Delta^{\prime}(f, X) \stackrel{j_{0}}{\longrightarrow} \pi_{0} \mathcal{S}^{\prime}(f, X) \stackrel{\rho}{\longrightarrow} G(f, X),
$$

see [15, Section 4]. From [12, Theorem 5.2], we have another short exact sequence

$$
\pi_{1} \mathcal{D}_{\text {id }}(M, X) \times \pi_{0} \Delta^{\prime}(f, X) \stackrel{\iota_{1}}{\longrightarrow} \pi_{1} \mathcal{O}_{f}(f, X) \stackrel{\rho \circ \partial_{1}}{\longrightarrow} G(f, X),
$$

in which $\iota_{1}$ is defined as follows. Let $\alpha \in \pi_{1} \mathcal{D}_{\text {id }}(M, X)$ be an element represented by some loop $\left\{h^{t}: M \rightarrow M\right\}_{t \in[0,1]}$ in $\mathcal{D}_{\text {id }}(M, X)$ such that 
$h^{0}=h^{1}=\operatorname{id}_{M}$, and $\phi \in \Delta^{\prime}(f, X)=\Delta(f) \cap \mathcal{D}_{\text {id }}(M, X)$. Fix any isotopy $\left\{\phi^{t}: M \rightarrow M\right\}_{t \in[0,1]}$ between $\operatorname{id}_{M}=\phi^{0}$ and $\phi=\phi^{1}$. Then

$$
\iota_{1}(\alpha, \phi)=\left[f \circ h^{t} \circ \phi^{t}\right] \in \pi_{1} \mathcal{O}_{f}(f, X) .
$$

1.3. Main diagram. Thus for a given Morse function $f$ on a smooth compact oriented surface $M$ we considered several spaces associated with $f$. If $M \neq S^{2}$, then all non-trivial homotopy information is encoded in the following commutative diagram:

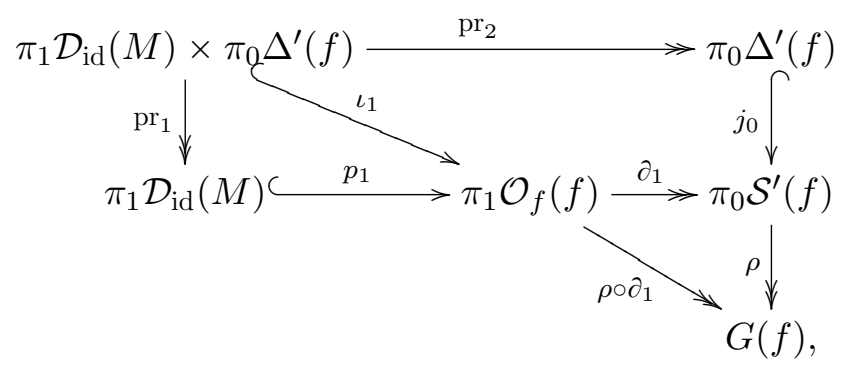

where $\operatorname{pr}_{1}$ and $\mathrm{pr}_{2}$ are projections onto the first and the second factor. In diagram (1.4) horizontal, vertical and diagonal sequences coincide with sequences (1.1), (1.2), and (1.3) respectively.

Let

$$
\begin{aligned}
& A_{1} \stackrel{i_{1}}{\longrightarrow} A_{2} \stackrel{p_{1}}{\longrightarrow} A_{3}, \\
& B_{1} \stackrel{i_{2}}{\longrightarrow} B_{2} \stackrel{p_{2}}{\longrightarrow} B_{3}
\end{aligned}
$$

be two exact sequences of groups. Recall that sequences (1.5) and (1.6) are isomorphic if there exist isomorphisms $\phi=\left\{\phi_{i}: A_{i} \rightarrow B_{i}, i=1,2,3\right\}$ such that the following diagram commutes

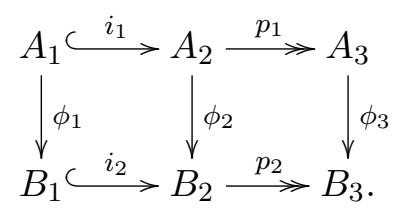

Similarly one can define the notion of an isomorphism for commutative diagrams.

The main aim of the paper is to describe diagram (1.4) for Morse functions on 2-torus up to an isomorphism.

1.4. Acknowledgments. The author is grateful to Sergiy Maksymenko for useful discussions. 
1.5. Structure of the paper. Section 2 collects definitions of wreath products, which we need to state our main result, Theorem 3.2. We recall some known results about Morse functions on 2-torus and their graphs in Section 4, and the fundamental groups of such functions is described in Section 6. Section 5 contains some facts needed for the proofs of our results, and we will prove Theorem 3.2 in Sections 7 and 8 .

\section{WREATH PRODUCTS}

To state our results we need special kinds of wreath products of groups with cyclic groups which we describe below. Let $G$ be a group and $n, m \geqslant 1$ be integers. We will consider the following wreath products:

- $G \imath_{n} \mathbb{Z}:=G^{n} \rtimes_{\alpha} \mathbb{Z}$,

- $G \nmid \mathbb{Z}_{n}:=G^{n} \rtimes_{\beta} \mathbb{Z}_{n}$,

- $G l_{n, m} \mathbb{Z}^{2}:=G^{n m} \rtimes_{\gamma} \mathbb{Z}^{2}$,

- $G$ ᄀ $\left(\mathbb{Z}_{n} \times \mathbb{Z}_{m}\right):=G^{n m} \rtimes_{\delta}\left(\mathbb{Z}_{n} \times \mathbb{Z}_{m}\right)$,

where $\alpha: G^{n} \times \mathbb{Z} \rightarrow G^{n}$ and $\beta: G \times \mathbb{Z}_{n} \rightarrow G^{n}$ correspond respectively to a non-effective $\mathbb{Z}$-action and an effective $\mathbb{Z}_{n}$-action on $G^{n}$ by cyclic shifts of coordinates defined by formulas:

$$
\left(\left(g_{i}\right)_{i=0}^{n-1}, a\right) \stackrel{\alpha}{\longmapsto}\left(g_{i+a}\right)_{i=0}^{n-1}, \quad\left(\left(g_{i}\right)_{i=0}^{n-1}, b\right) \stackrel{\beta}{\longmapsto}\left(g_{i+b}\right)_{i=0}^{n-1},
$$

where all indexes are taken modulo $n, g_{i} \in G, a \in \mathbb{Z}, b \in \mathbb{Z}_{n}$. Similarly $\gamma: G^{n m} \times \mathbb{Z}^{2} \rightarrow G^{n m}$ and $\delta: G^{n m} \times\left(\mathbb{Z}_{n} \times \mathbb{Z}_{m}\right) \rightarrow G^{n m}$ correspond respectively to a non-effective $\mathbb{Z}^{2}$-action and an effective $\mathbb{Z}_{n} \times \mathbb{Z}_{m}$-action on $G^{n m}$ by cyclic shifts of the corresponding coordinates defined by formulas

$$
\begin{gathered}
\left(\left(g_{i j}\right)_{i, j=0}^{n-1, m-1},(a, b)\right) \stackrel{\gamma}{\longmapsto}\left(g_{i+a, j+b}\right)_{i, j=0}^{n-1, m-1}, \\
\left(\left(g_{i j}\right)_{i, j=0}^{n-1, m-1},\left(a^{\prime}, b^{\prime}\right)\right) \stackrel{\delta}{\longmapsto}\left(g_{i+a^{\prime}, j+b^{\prime}}\right)_{i, j=0}^{n-1, m-1},
\end{gathered}
$$

where the indexes $i$ and $j$ takes modulo $n$ and $m$ respectively, $(a, b) \in \mathbb{Z}^{2}$, $\left(a^{\prime}, b^{\prime}\right) \in \mathbb{Z}_{n} \times \mathbb{Z}_{m}$.

So $G \imath_{n} \mathbb{Z}$ and $G \imath \mathbb{Z}_{n}$ are direct products of sets $G^{n} \times \mathbb{Z}$ and $G^{n} \times \mathbb{Z}_{n}$ with the following multiplications

$$
(g, a) \cdot\left(g^{\prime}, a^{\prime}\right)=\left(\alpha\left(g, a^{\prime}\right) g^{\prime}, a+a^{\prime}\right), \quad(g, a) \cdot\left(g^{\prime}, a^{\prime}\right)=\left(\beta\left(g, b^{\prime}\right) g^{\prime}, b+b^{\prime}\right),
$$

for $g, g^{\prime} \in G^{n}, a, a^{\prime} \in \mathbb{Z}$, and $b, b^{\prime} \in \mathbb{Z}_{n}$. Similarly $G \imath_{n, m} \mathbb{Z}^{2}$ and $G$ ? $\left(\mathbb{Z}_{n} \times \mathbb{Z}_{m}\right)$ are direct products of sets $G^{n m} \times \mathbb{Z}^{2}$ and $G^{n m} \times \mathbb{Z}_{n} \times \mathbb{Z}_{m}$ respectively with multiplications

$$
\begin{aligned}
& (g,(a, b)) \cdot\left(g^{\prime},\left(a^{\prime}, b^{\prime}\right)\right)=\left(\gamma\left(g, a^{\prime}, b^{\prime}\right) g^{\prime},\left(a+a^{\prime}, b+b^{\prime}\right)\right) \\
& (g,(c, d)) \cdot\left(g^{\prime},\left(c^{\prime}, d^{\prime}\right)\right)=\left(\gamma\left(g, c^{\prime}, d^{\prime}\right) g^{\prime},\left(c+c^{\prime}, d+d^{\prime}\right)\right)
\end{aligned}
$$


for $g, g^{\prime} \in G^{n m}, a, a^{\prime} b, b^{\prime} \in \mathbb{Z}, c, c^{\prime} \in \mathbb{Z}_{n}$, and $d, d^{\prime} \in \mathbb{Z}_{m}$.

The general definition of wreath product and its properties the reader can find in [18].

\section{MAin RESUlt}

Let $f$ be a Morse function on $T^{2}, \Gamma_{f}$ be its graph, and $p_{f}: T^{2} \rightarrow \Gamma_{f}$ be the projection map induced by $f$.

Lemma 3.1. The map $p_{f}^{*}: \pi_{1} T^{2} \rightarrow \pi_{1} \Gamma_{f}$ induced by $p_{f}$ is an epimorphism with a nonzero kernel. Hence the graph of $f$ is either a tree, or has a unique circuit.

Proof. Let $x \in T^{2}$ be an arbitrary point. Note that $\pi_{1}\left(\Gamma_{f}, p_{f}(x)\right)=F_{k}$, where $F_{k}$ is a free group of some rank $k$ equals the number of independent cycles in $\Gamma_{f}$, and $\pi_{1}\left(T^{2}, x\right)$ is isomorphic to $\mathbb{Z}^{2}$. It is easy to see that there exists a map $s:\left(\Gamma_{f}, p_{f}(x)\right) \rightarrow\left(T^{2}, x\right)$ such that the composition $p_{f} \circ s$ is homotopic to the identity map $\operatorname{id}_{\Gamma_{f}}$ relatively base point. Then the composition

$$
\pi_{1}\left(\Gamma_{f}, p_{f}(x)\right) \stackrel{s *}{\longrightarrow} \pi_{1}\left(T^{2}, x\right) \stackrel{p_{f}^{*}}{\longrightarrow} \pi_{1}\left(\Gamma_{f}, p_{f}(x)\right)
$$

is the identity isomorphism.

Moreover $p_{f}^{*}$ is a surjective map and $\pi_{1}\left(\Gamma_{f}, p_{f}(x)\right)=F_{k}$ is a subgroup of the group $\pi_{1}\left(T^{2}, x\right)=\mathbb{Z}^{2}$. Since $\pi_{1}\left(T^{2}, x\right)$ is commutative, it follows that $F_{k}$ as a subgroup of $\pi_{1}\left(T^{2}, x\right)$ is also a commutative group. This is possible only when $k=0$ or $k=1$. So $\Gamma_{f}$ is either a tree if $k=0$, or $\Gamma_{f}$ has a unique circuit if $k=1$. In both of these cases $p_{f}^{*}$ has a nonzero kernel.

Denote by $\mathscr{F}_{0}$ the class of Morse functions on $T^{2}$ whose graphs are trees, and by $\mathscr{F}_{1}$ the class of Morse functions on $T^{2}$ whose graphs contain circuits ${ }^{1}$.

Our main result is the following theorem.

Theorem 3.2. Let $f$ be a Morse function on $T^{2}$ and $\Gamma_{f}$ be its graph.

(1) Assume that $f$ belongs to $\mathscr{F}_{0}$. Then there exists a set of mutually disjoint 2 -disks $\mathbb{D}=\left\{D_{i}\right\}_{i=1}^{r} \subset T^{2}$ for some $r \geqslant 1$ such that each restriction $\left.f\right|_{D_{i}}: D_{i} \rightarrow \mathbb{R}, i=1, \ldots, r$, is also a Morse function, and the diagram (1.4) is isomorphic to

\footnotetext{
${ }^{1}$ Thus the index $i$ in $\mathscr{F}_{i}$ refers to the rank of homology group $H_{1}\left(\Gamma_{f}, \mathbb{Z}\right)$.
} 


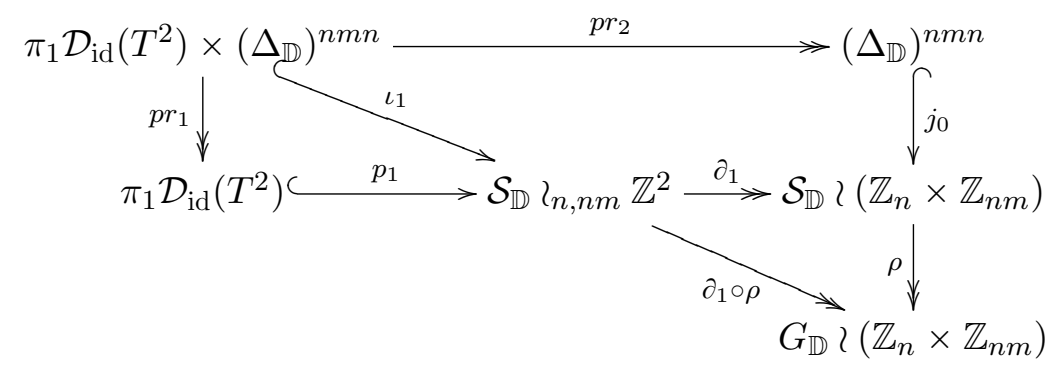

for some $n, m \in \mathbb{N}$, where

$$
\Delta_{\mathbb{D}}=\prod_{i=1}^{r} \pi_{0} \Delta^{\prime}\left(\left.f\right|_{D_{i}}, \partial D_{i}\right), \quad \mathcal{S}_{\mathbb{D}}=\prod_{i=1}^{r} \pi_{0} \mathcal{S}^{\prime}\left(\left.f\right|_{D_{i}}, \partial D_{i}\right), \quad G_{\mathbb{D}}=\prod_{i=1}^{r} G\left(\left.f\right|_{D_{i}}\right) .
$$

(2) Assume that $f$ belongs to $\mathscr{F}_{1}$. Then there exists a set of mutually disjoint 2-disks $\mathbb{Y}=\left\{Y_{i j}\right\}_{i=0, \ldots, k}^{j=0, \ldots, c_{i}} \subset T^{2}$ for certain $k, c_{i} \in \mathbb{N}$, and $m_{i} \in \mathbb{N}$, $i=0, \ldots, k-1$, such that diagram (1.4) is isomorphic to

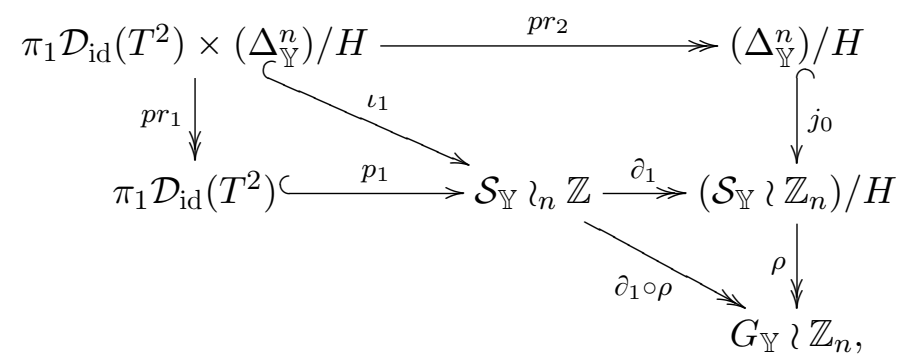

for some $n \in \mathbb{N}$, where

$$
\begin{array}{rlrl}
\Delta_{\mathbb{Y}} & =\prod_{i=1}^{k}\left(\left(\prod_{j=1}^{c_{i}} \Delta_{Y_{i j}}\right)^{m_{i}} \times m_{i} \mathbb{Z}\right), & \Delta_{Y_{i j}}=\pi_{0} \Delta^{\prime}\left(\left.f\right|_{Y_{i j}}, \partial Y_{i j}\right), \\
\mathcal{S}_{\mathbb{Y}}=\prod_{i=1}^{k}\left(\left(\prod_{j=1}^{c_{i}} \mathcal{S}_{Y_{i j}}\right)_{2_{m_{i}}} \mathbb{Z}\right), & \mathcal{S}_{Y_{i j}}=\pi_{0} \mathcal{S}^{\prime}\left(\left.f\right|_{Y_{i j}}, \partial Y_{i j}\right), \\
G_{\mathbb{Y}}=\prod_{i=1}^{k}\left(\left(\prod_{j=1}^{c_{i}} G_{Y_{i j}}\right) \backslash \mathbb{Z}_{m_{i}}\right), & G_{Y_{i j}}=G\left(\left.f\right|_{Y_{i j}}, \partial Y_{i j}\right),
\end{array}
$$

and the group $H$ is a normal subgroup of $\Delta_{\mathbb{Y}}^{n}$ isomorphic to $\mathbb{Z}$ and generated by the element

$$
(\underbrace{\left(E_{1}, m_{1}, E_{2}, m_{2}, \ldots, E_{k}, m_{k}\right), \ldots,\left(E_{1}, m_{1}, E_{2}, m_{2}, \ldots, E_{k}, m_{k}\right)}_{n}),
$$


where $E_{i}$ is the unit of the group $\left(\prod_{j=1}^{c_{i}} \Delta_{Y_{i j}}\right)^{m_{i}}, i=0,1 \ldots, k$. The group $H$ is contained in the center of $\mathcal{S}_{\mathbb{Y}} 2_{n} \mathbb{Z}$ via $j_{0}(h)=(h, 0)$ for $h \in H$.

Remark 3.3. (1) It is known that the inclusion $T^{2} \hookrightarrow \mathcal{D}_{\text {id }}\left(T^{2}\right)$ is a homotopy equivalence, see $[1,3]$. So, the group $\pi_{1} \mathcal{D}_{\text {id }}\left(T^{2}\right)$ is isomorphic to $\mathbb{Z}^{2}$.

(2) S. Maksymenko showed that the group $\pi_{1} \mathcal{O}_{f}(f)$ is a subgroup of the braid group of $T^{2}$, see for example [15, Theorem 1.1]. The element (3.2) can be regarded as a certain analogue of the Garside element in this braid group.

(3) In a series of papers $[17,9,16,2]$ S. Maksymenko and the author described groups $\pi_{1} \mathcal{O}_{f}(f)$ for functions on 2-torus. We shortly review these results in Section 6 .

(4) Since the structure of groups $\pi_{1} \mathcal{D}_{\text {id }}\left(T^{2}\right)$ and $\pi_{1} \mathcal{O}_{f}(f)$ are known, it follows that for description of diagram (1.4) we need to find a structure of groups $\pi_{0} \mathcal{S}^{\prime}(f), G(f)$, and $\pi_{0} \Delta^{\prime}(f)$.

4. Functions on 2-torus, their GRAPHS, AND homotopy PROPERTIES

In this section we give a short overview of known results about graphs of smooth functions on 2-torus, see $[9,17,16,2]$. Let $f$ be a Morse function on $T^{2}, \Gamma_{f}$ be its graph, and $p_{f}: T^{2} \rightarrow \Gamma_{f}$ be the projection map induced by $f$.

Case 1: Functions from $\mathscr{F}_{0}$. The following lemma holds.

Lemma 4.1. [9, Proposition 1]. Let $f \in \mathscr{F}_{0}$. Then there exits a unique vertex $v$ in $\Gamma_{f}$ such that each component of the complement $T^{2} \backslash p_{f}^{-1}(v)$ is an open 2-disk. Such a vertex $v$ of $\Gamma_{f}$ is called special.

Let $v$ be a special vertex of $\Gamma_{f}, G_{v}$ be the stabilizer of $v$ with respect to the action of the group $G(f)$ acting on $\Gamma_{f}$. Then a uniqueness of the special vertex $v$ implies that $G_{v}$ coincides with $G(f)$. Let st $(v)$ be a $G_{v}$-invariant connected neighborhood of $v$ containing no other vertices of $\Gamma_{f}$. The set $G_{v}^{l o c}=\left\{\left.g\right|_{\operatorname{st}(v)} \mid g \in G_{v}\right\}$ consisting of restrictions of elements of $G_{v}$ onto $\operatorname{st}(v)$ is a subgroup of $\operatorname{Aut}(\operatorname{st}(v))$. We will call $G_{v}^{l o c}$ the local stabilizer of $v$. Let also $r: G_{v} \rightarrow G_{v}^{l o c}$ be the restriction map to $\operatorname{st}(v)$.

Lemma 4.2. [2, Theorem 2.5]. Let $f \in \mathscr{F}_{0}$ and $v$ be the special vertex of $\Gamma_{f}$.

(1) Then $G_{v}^{\text {loc }}$ is isomorphic to $\mathbb{Z}_{n} \times \mathbb{Z}_{n m}$ for some $n, m \in \mathbb{N}$.

(2) There exists a section $s: G_{v}^{\text {loc }} \rightarrow \mathcal{S}^{\prime}(f)$ of the map $r \circ \rho$ such that $s\left(G_{v}^{\text {loc }}\right)$ freely acts on $T^{2}$, so the map $q: T^{2} \rightarrow T^{2} / s\left(G_{v}^{\text {loc }}\right)$ is a covering 
projection. Moreover the space of orbits $T^{2} / s\left(G_{v}^{l o c}\right)$ is also diffeomorphic to $T^{2}$.

Remark 4.3. (i) Throughout the paper by $G_{v}^{l o c}$-action on $T^{2}$ we will mean its action via the section $s$ from (2). So we will write $T^{2} / G_{v}^{l o c}$ instead of $T^{2} / s\left(G_{v}^{l o c}\right)$.

(ii) Let $V=p_{f}^{-1}(v)$ be a critical component of a level set of $f$ which corresponds to the special vertex $v$. Since $G_{v}^{l o c}$ freely acts on $T^{2}$, it follows that connected components of $T^{2} \backslash p_{f}^{-1}(v)$ can be enumerated by three indexes $D_{i j k}, i=1,2, \ldots, r, j=0, \ldots, n-1$, and $k=0, \ldots, n m-1$. So, if $\gamma=(a, b) \in G_{v}^{l o c}=\mathbb{Z}_{n} \times \mathbb{Z}_{n m}$ then

$$
\gamma\left(D_{i j k}\right)=D_{i, j+a \bmod n, k+b \bmod n m} .
$$

(iii) The set $\mathbb{D}=\left\{D_{i 00}\right\}_{i=1}^{r}$ from (1) of Theorem 3.2 will be called a fundamental set of this $G_{v}^{l o c}$-action. Numbers $n, m$ and $r$ in (1) of Theorem 3.2 are also determined from (i).

Case 2: Functions from $\mathscr{F}_{1}$. Let $f \in \mathscr{F}_{1}, \Theta$ be a unique simple cycle in $\Gamma_{f}, z \in \Theta$ be a point belonging to some edge of $\Theta$, and $C_{0}=p_{f}^{-1}(z) \subset T^{2}$ be the corresponding regular component of some level set $f^{-1}(c), c \in \mathbb{R}$.

Notice that $f^{-1}(c)$ consists of finitely many, say $n$, connected components and is invariant under the action of any $h \in \mathcal{S}(f)$. Let

$$
\mathcal{C}=\left\{h\left(C_{0}\right) \mid h \in \mathcal{S}^{\prime}(f)\right\}
$$

be the set of all images of $C_{0}$ under the action of elements from $\mathcal{S}^{\prime}(f)$. Evidently, the curves from $\mathcal{C}$ are pairwise disjoint. Since $C_{0}$ does not separate $T^{2}$, it follows that each $C_{i}$ does not separate $T^{2}$ as well. Two such curves will be called parallel, and the set $\mathcal{C}$ will be called parallel family on $T^{2}$.

We can also assume that they are cyclically enumerated along $T^{2}$, so that $C_{i}$ and $C_{i+1}$ bound a cylinder $Q_{i}$ such that the interior of $Q_{i}$ does not intersect $\mathcal{C}$, where all indexes are taken modulo $n$. The number $n$ from (2) of Theorem 3.2 is the number of curves in $\mathcal{C}$ and we will call it the cyclic index of $f$. For other details see [16].

\section{Preliminaries}

5.1. Homotopy properties of Morse functions on cylinder. The following lemma holds.

Lemma 5.2. [15, Theorem 5.8, Lemma 5.1 III (a)]. Let $f$ be a Morse function on cylinder $Q=S^{1} \times[0,1]$. Then there exists a family of mutually disjoint 2-disks $\mathbb{Y}=\left\{Y_{i j}\right\}_{i=1, \ldots, k}^{j=1, \ldots, c_{i}} \subset Q$ for some $k, c_{i} \in \mathbb{N}$, and $m_{i} \in \mathbb{N}$, $i=1, \ldots, k$ such that 
(1) $\left.f\right|_{Y_{i j}}$ is also a Morse function for each $j=1, \ldots, c_{i}, i=1, \ldots, k$;

(2) there exists an isomorphism $\zeta=\left(\zeta_{1}, \zeta_{2}, \zeta_{3}\right)$ of the following short exact sequences:

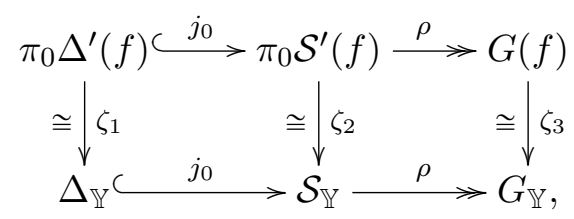

where the first row is a sequence (1.2) with $X=\varnothing$, and

$$
\begin{array}{rlrl}
\Delta_{\mathbb{Y}} & =\prod_{i=1}^{k}\left(\left(\prod_{j=1}^{c_{i}} \Delta_{Y_{i j}}\right)^{m_{i}} \times m_{i} \mathbb{Z}\right), & \Delta_{Y_{i j}}=\pi_{0} \Delta^{\prime}\left(\left.f\right|_{Y_{i j}}, \partial Y_{i j}\right), \\
\mathcal{S}_{\mathbb{Y}}=\prod_{i=1}^{k}\left(\left(\prod_{j=1}^{c_{i}} \mathcal{S}_{Y_{i j}}\right) \imath_{m_{i}} \mathbb{Z}\right), & \mathcal{S}_{Y_{i j}}=\pi_{0} \mathcal{S}^{\prime}\left(\left.f\right|_{Y_{i j}}, \partial Y_{i j}\right), \\
G_{\mathbb{Y}}=\prod_{i=1}^{k}\left(\left(\prod_{j=1}^{c_{i}} G_{Y_{i j}}\right) \mathbb{Z}_{m_{i}}\right), & G_{Y_{i j}}=G\left(\left.f\right|_{Y_{i j}}, \partial Y_{i j}\right) .
\end{array}
$$

(3) The kernel of the homomorphism $\pi_{0} \mathcal{S}^{\prime}(f, \partial Q) \rightarrow \pi_{0} \mathcal{S}^{\prime}(f)$ induced by the inclusion is isomorphic to $\mathbb{Z}$ and is generated by the isotopy class of $\tau_{0} \circ \tau_{1}^{-1}$, where $\tau_{i} \in S^{\prime}(f)$ is a Dehn twist along $S^{1} \times\{i\}, i=0,1$. Moreover, in the notation of (2) $\left[\tau_{0} \circ \tau_{1}^{-1}\right]$ corresponds to the element

$$
\left(E_{1}, m_{1}, \ldots, E_{k}, m_{k}\right) \in \prod_{i=1}^{k}\left(\left(\prod_{j=1}^{c_{i}} \Delta_{Y_{i j}}\right)^{m_{i}} \times m_{i} \mathbb{Z}\right),
$$

where $E_{i}$ is the unit of $\left(\prod_{j=1}^{c_{i}} \Delta_{Y_{i j}}\right)^{m_{i}}, i=1, \ldots, k$, [15, Lemma 5.1 III (a)].

5.3. Dehn twists and slides. Let $\alpha, \beta:[-1,1] \rightarrow[0,1]$ be two smooth functions such that $\alpha=0$ on $[-1,-1 / 2]$ and $\alpha=1$ on $[1 / 2,1]$, while $\beta=0$ on $[-1,-2 / 3] \cup[2 / 3,1]$, and $\beta=1$ on $[-1 / 3,1 / 3]$.

Let $Q=S^{1} \times[-1,1]$ be a cylinder and $C=S^{1} \times 0$. Define the following two diffeomorphisms of $Q$ by the formulas

$$
\tau(z, t)=\left(z e^{\alpha(t)}, t\right), \quad \theta(z, t)=\left(z e^{\beta(t)}, t\right),
$$

for $(z, t) \in S^{1} \times[-1,1]$. The diffeomorphisms $\tau$ and $\theta$ are called a Dehn twist and a slide along the curve $C$ respectively. Note that $\tau$ is fixed on some neighborhood of $\partial Q$ and $\theta$ is fixed on some neighborhood of $C \cup \partial Q$.

Let $M$ be a smooth surface and $C \subset M$ be a simple closed curve. Suppose that $C$ is a two-sided curve, i.e., $C$ has a regular neighborhood $W$ diffeomorphic to a cylinder $Q$. Fix any diffeomorphism $\phi: Q \rightarrow W$ such 
that $\phi\left(S^{1} \times 0\right)=C$. Since $\tau$ is fixed on some neighborhood of $\partial Q$, it is easy to see that $\phi \circ \tau \circ \phi^{-1}: W \rightarrow W$ extends by the identity to a unique diffeomorphism $\bar{\tau}$ of $M$. Any diffeomorphism $h: M \rightarrow M$ isotopic to $\bar{\tau}$ or $\bar{\tau}^{-1}$ will be called a Dehn twist along $C$.

Similarly $\phi \circ \theta \circ \phi^{-1}: W \rightarrow W$ extends by the identity map to the diffeomorphism $\bar{\theta}$ of $M$. Any diffeomorphism $h: M \rightarrow M$ fixed on some neighborhood of $C$ supported on some cylindrical neighborhood $W$ of $C$ and isotopic to $\bar{\theta}$ or $\bar{\theta}^{-1}$ relatively to some neighborhood of $C \cup \overline{M \backslash Q}$ will be called a slide along $C$. For more details see [16].

5.4. Groups $\pi_{0} \Delta^{\prime}(f)$ and their special subgroups. Let $f$ be a Morse function from $\mathscr{F}_{1}$ with cyclic index $n$, so there exists an $\mathcal{S}^{\prime}(f)$-invariant family

$$
\mathcal{C}=\left\{C_{i} \mid i=0, \ldots, n-1\right\}
$$

of connected components of the same level set of $f$ and neither of those curves separate $T^{2}, i=0, \ldots, n-1$. We let denote by $Q_{i}$ the cylinder bounded by curves $C_{i}$ and $C_{i+1} \bmod n$.

A neighborhood $V$ of $C \in \mathcal{C}$ will be called $f$-adapted if

- $V$ is diffeomorphic to $S^{1} \times[0,1]$ via a diffeomorphism, say $\phi$, such that - $\phi^{-1}\left(S^{1} \times\{t\}\right)$ is a connected component of some level set of $f, t \in[0,1]$.

In particular, $V$ does not contain critical points of $f$. Fix an $f$-adapted neighborhood $V_{i}$ of $C_{i}, i=0, \ldots, n-1$, so that

- $V_{i} \cap V_{j}=\varnothing$ for $i \neq j$;

- for each $i, j$ there exists $h \in \mathcal{S}^{\prime}(f)$ such that $h\left(V_{i}\right)=V_{j}$, so the union

$$
\mathrm{V}=\bigcup_{i=0}^{n-1} V_{i}
$$

is $\mathcal{S}^{\prime}(f)$-invariant.

Notice that by definition the group $\Delta^{\prime}(f, \mathrm{~V})$ consists of diffeomorphisms of $T^{2}$ which are isotopic to $\mathrm{id}_{T^{2}}$, fixed on an $f$-adapted neighborhood $\mathrm{V}$ of $\mathcal{C}$, and inducing trivial homeomorphisms of $\Gamma_{f}$. Let $j: \Delta^{\prime}(f, \mathrm{~V}) \rightarrow \Delta^{\prime}(f)$ be the natural inclusion. It is known and is easy to see that the homomorphism

$$
j_{0}: \pi_{0} \Delta^{\prime}(f, \mathrm{~V}) \rightarrow \pi_{0} \Delta(f)
$$

induced by $j$ is an epimorphism, see [15, Lemma 5.1]. Let also $W_{i}$ be an $f$-adapted neighborhood of $C_{i}$ satisfying $V_{i} \subset \operatorname{Int} W_{i}$ for $i=0,1 \ldots, n-1$. Put $\mathrm{W}=\bigcup_{i=0}^{n-1} W_{i}$, Then, [15, Corollary 7.2], the natural inclusion

$$
\Delta^{\prime}(f, \mathrm{~V}) \hookrightarrow \Delta^{\prime}(f, \mathrm{~W})
$$

is the homotopy equivalence and groups $\pi_{0} \Delta^{\prime}(f)$ and $\pi_{0} \Delta^{\prime}(f, \mathrm{~V})$ are abelian groups. 
Recall that a vector field $F$ on a smooth oriented surface $M$ is called Hamiltonian-like for a Morse function $f$ if the following conditions hold:

- singular points of $F$ correspond to critical points of $f$,

- $f$ is constant along $F$,

- for every critical point $z$ of $f$ there exists a local coordinate system $(x, y)$ such that $z=(0,0), f(x, y)= \pm x^{2} \pm y^{2}+f(z)$ near $z$, and in these coordinates $F$ has the form $F(x, y)=-f_{y}^{\prime} \frac{\partial}{\partial x}+f_{x}^{\prime} \frac{\partial}{\partial y}$.

By [10, Lemma 5.1] for every Morse function $f: M \rightarrow \mathbb{R}$ on a smooth oriented surface $M$ there exists a Hamiltonian-like vector field.

Fix a Hamiltonian-like vector field $F$ for the given function Morse function $f$ on $T^{2}$, and let $\mathbf{F}$ be the flow of $F$. Then $\mathbf{W}$ is $\mathbf{F}$-invariant and consists of periodic orbits. Therefore one can assume that periods of all trajectories of $\mathbf{F}$ in $\mathbf{W}$ equals 1 .

Let $\theta_{i}: T^{2} \rightarrow T^{2}$ be a slide along $C_{i}$ supported in $W_{i} \backslash V_{i}, i=0, \ldots, n-1$, and

$$
\theta=\theta_{0} \circ \theta_{1} \circ \ldots \circ \theta_{n-1} .
$$

Then, [16, Lemma 5.2], there exists a smooth function $\sigma: T^{2} \rightarrow \mathbb{R}$ such that

- $\sigma$ is constant along trajectories of $\mathbf{F}$,

- $\sigma=1$ on $\mathrm{V}, \sigma=0$ on $T^{2} \backslash \mathrm{W}$, and

- $\theta=\mathbf{F}_{\sigma}$.

Then for $k \in \mathbb{Z}$ we have $\theta^{k}=\mathbf{F}_{k \sigma}$. From this definition it immediately follows that $\theta$ belongs to $\Delta^{\prime}(f, \mathrm{~V})$. A free abelian subgroup of $\pi_{0} \Delta^{\prime}(f, \mathrm{~V})$ generated by $\theta$ will be denoted by $\langle\theta\rangle$. The following theorem is our main result of this section.

Theorem 5.5. For $f \in \mathscr{F}_{1}$ the following statements hold true.

1) $\operatorname{Ker} j_{0} \cong\langle\theta\rangle \cong \mathbb{Z}$. In other words, each $h \in \Delta^{\prime}(f, \mathrm{~V})$ is isotopic to $\theta^{k(h)}$ for some $k(h) \in \mathbb{Z}$ relatively to $\mathrm{V}$ whenever $[h] \in \operatorname{Ker} j_{0}$.

2) The following exact sequence of abelian groups

$$
\langle\theta\rangle \bigodot \pi_{0} \Delta^{\prime}(f, \mathrm{~V}) \stackrel{j_{0}}{\longrightarrow} \pi_{0} \Delta^{\prime}(f)
$$

splits. In particular, there is an isomorphism

$$
\pi_{0} \Delta^{\prime}(f, \mathrm{~V}) \cong\langle\theta\rangle \times \pi_{0} \Delta^{\prime}(f) .
$$

Proof. 1) Let $h \in \Delta^{\prime}(f, \mathrm{~V})$. We have to show that if $[h] \in \operatorname{Ker} j_{0}$, then $h$ is isotopic to $\theta^{k(h)}$ relatively $\mathrm{V}$ for some $k(h) \in \mathbb{Z}$.

Recall that the identity component of $\Delta^{\prime}(f)$ is $\mathcal{S}_{\text {id }}(f)$, see [15, Lemma 4.1]. Hence if $h \in \Delta^{\prime}(f, \mathrm{~V})$ is such that $[h] \in \operatorname{Ker} j_{0}$, then $h \in \mathcal{S}_{\text {id }}(f)$, and 
by [15, Lemma 6.1] there exists a unique smooth function $\alpha: T^{2} \rightarrow \mathbb{R}$ such that $h=\mathbf{F}_{\alpha}$.

We claim that such $h$ admits some "simplification" on $T^{2} \backslash \mathrm{W}$, i.e., $h$ can be deformed relatively $\mathrm{V}$ to a diffeomorphism $h^{\prime}$ such that $\left[h^{\prime}\right] \in \operatorname{Ker}\left(j_{0}\right)$ and $h^{\prime}$ is also fixed on $T^{2} \backslash \mathrm{W}$. Indeed, fix any smooth function $\delta: T^{2} \rightarrow \mathbb{R}$ satisfying

- $\left.\delta\right|_{\mathrm{V}}=0,\left.\delta\right|_{T^{2} \backslash \mathrm{W}}=1$,

- $\delta$ is constant along trajectories of $\mathbf{F}$,

and define the following homotopy $H^{t}: T^{2} \rightarrow T^{2}, t \in[0,1]$, by the formula

$$
H^{t}(h)=\mathbf{F}_{t \delta \alpha}^{-1} \circ h .
$$

By [15, Lemma 6.1] a family $\left\{H^{t}\right\}$ is in fact an isotopy between $H^{0}=h$, $h^{\prime}:=H^{1}(h)=\mathbf{F}_{\delta \alpha}^{-1} \circ h$, and each diffeomorphism $H^{t}(h)$ belongs to $\Delta^{\prime}(f, \mathrm{~V})$. In particular, the isotopy classes of $h$ and $h^{\prime}$ in $\pi_{0} \Delta^{\prime}(f, \mathrm{~V})$ coincide, and $h^{\prime}$ is fixed on $T^{2} \backslash \mathrm{W}$.

So we can redenote $h^{\prime}$ with $h$ and additionally assume further that $h$ is fixed on $T^{2} \backslash \mathrm{W}$. The restriction of $\alpha$ and $h$ onto $V_{i}, i=0, \ldots, n-1$, will also be denoted by $\alpha_{i}$ and $h_{i}$ respectively. By assumptions $h$ is fixed on $\mathrm{V}$, that is

$$
h_{i}(x)=\mathbf{F}_{\alpha_{i}(x)}(x)=x, \quad x \in V_{i} .
$$

Since the period of trajectories of $\mathbf{F}$ on $\mathbf{W}$ equals 1 , it follows that $\alpha_{i}$ takes an integer value, say $k_{i}(h) \in \mathbb{Z}$, depending on $h$ for each $x \in V_{i}$.

We claim that in fact $\alpha_{i}$ takes the same value $k \in \mathbb{Z}$ on each $V_{i}$, so all of those numbers $k_{i}(h)$ must coincide. Indeed, recall that $\left.h\right|_{Q_{i}}$ is isotopic relatively $\mathrm{V} \cap Q_{i}$ to a Dehn twist $\tau^{a_{i}}$ supported in $\mathrm{V} \cap Q_{i}$, where

$$
a_{i}=\alpha\left(C_{i+1}\right)-\alpha\left(C_{i}\right)=k_{i+1}(h)-k_{i}(h), \quad i=0, \ldots, n-1 .
$$

However, as $h \in \Delta^{\prime}(f, \mathrm{~V})$, it follows that $\left.h\right|_{Q_{i}}, i=0, \ldots, n-1$, is isotopic to $\operatorname{id}_{Q_{i}}=\tau^{0}$ relatively to $\mathrm{V} \cap Q_{i}$, that is

$$
k_{i+1}(h)-k_{i}(h)=a_{i}=0,
$$

and so $k_{i+1}(h)=k_{i}(h)$ for each $i=0, \ldots, n-1$. Thus we can denote the common value of all $k_{i}(h)$ simply by $k(h)$.

Define now an isotopy $H^{t}(h): T^{2} \rightarrow T^{2}$ between $h=\mathbf{F}_{\alpha}$ and $\theta^{k}=\mathbf{F}_{k(h) \sigma}$ by the formula

$$
H^{t}(h)=\mathbf{F}_{(1-t) \alpha+t k(h) \sigma}, \quad t \in[0,1] .
$$

Then $H^{t}(h)$ is fixed on $\vee$ for all $t \in[0,1]$ and so each $h$ with $[h] \in \operatorname{Ker} j_{0}$ is isotopic to $\theta^{k(h)}$ relatively to $\mathrm{V}$. In other words, $\operatorname{Ker} j_{0} \subset\langle\theta\rangle$.

The inverse inclusion is easy $\langle\theta\rangle \subset \operatorname{Ker} j_{0}$, and so $\operatorname{Ker} j_{0}=\langle\theta\rangle$ is a free abelian group generated by the element $\theta$. 
2) Now it follows from 1) and surjectivity of $j_{0}$ that we have a short exact sequence (5.2):

$$
\langle\theta\rangle \leftrightharpoons \pi_{0} \Delta^{\prime}(f, \mathrm{~V}) \stackrel{j_{0}}{\longrightarrow} \pi_{0} \Delta^{\prime}(f) .
$$

Moreover, [10], it consists of abelian groups and the group $\pi_{0} \Delta^{\prime}(f)$ is a free abelian. Therefore the above sequence splits, i.e., there is an isomorphism $\pi_{0} \Delta^{\prime}(f, \mathrm{~V}) \cong \pi_{0} \Delta^{\prime}(f) \times\langle\theta\rangle$.

\section{Orbits of Morse functions on 2-torus}

The following theorem describes the fundamental groups of orbits of Morse functions on 2-torus. We showed that they can be computed using zero homotopy groups of stabilizers of restrictions of the given function onto subsurfaces of $T^{2}$ being 2-disks and cylinders.

Theorem 6.1. $[17,9,16,2]$. Let $f$ be a Morse function on $T^{2}$, and $\Gamma_{f}$ be its graph.

(1) Assume that $f$ belongs to $\mathscr{F}_{0}$ and $G_{v}^{\text {loc }} \cong \mathbb{Z}_{n} \times \mathbb{Z}_{n m}$ for some $n, m \in \mathbb{N}$. Then there exists a set of mutually disjoint 2-disks $\mathbb{D}=\left\{D_{i}\right\}_{i=1}^{r} \subset T^{2}$ for some $r \in \mathbb{N}$ which is a fundamental set of the free $G_{v}^{\text {loc }}$-action on $T^{2}$ such that the restriction $\left.f\right|_{D_{i}}, i=1, \ldots, r$, is a Morse function, and there is an isomorphism

$$
\xi_{1}: \mathcal{S}_{\mathbb{D}} \imath_{n, n m} \mathbb{Z}^{2} \rightarrow \pi_{1} \mathcal{O}_{f}(f),
$$

where $\mathcal{S}_{\mathbb{D}}:=\prod_{i=1}^{r} \pi_{0} \mathcal{S}^{\prime}\left(\left.f\right|_{D_{i}}, \partial D_{i}\right)$. Moreover $r$ is the number of orbits of free $G_{v}^{\text {loc }}$-action on $T^{2}$.

(2) Assume that $f$ belongs to $\mathscr{F}_{1}$ and has a cyclic index $n \in \mathbb{N}$. Then there exists a cylinder $Q \subset T^{2}$ such that $\left.f\right|_{Q}$ is also a Morse function and we have an isomorphism

$$
\xi_{2}: \mathcal{S}_{Q} 2_{n} \mathbb{Z} \rightarrow \pi_{1} \mathcal{O}_{f}(f),
$$

where $\mathcal{S}_{Q}:=\pi_{0} \mathcal{S}^{\prime}\left(\left.f\right|_{Q}, \partial Q\right)$.

6.2. Strategy of the proof of Theorem 3.2. First we recall explicit definitions of isomorphisms

$$
\xi_{1}: \mathcal{S}_{\mathbb{D}} \imath_{n, n m} \mathbb{Z}^{2} \rightarrow \pi_{1} \mathcal{O}_{f}(f), \quad \xi_{2}: \mathcal{S}_{Q} \imath_{n} \mathbb{Z} \rightarrow \pi_{1} \mathcal{O}_{f}(f)
$$

from Theorem 6.1 in Subsections 7.1 and 8.1 respectively. Since groups $\pi_{0} \mathcal{S}^{\prime}(f)$ and $G(f)$ are quotient-groups

$$
\begin{gathered}
\pi_{0} \mathcal{S}^{\prime}(f) \cong \pi_{1} \mathcal{O}_{f}(f) / \pi_{1} \mathcal{D}_{\mathrm{id}}\left(T^{2}\right), \\
G(f) \cong \pi_{1} \mathcal{O}_{f}(f) /\left(\pi_{1} \mathcal{D}_{\mathrm{id}}\left(T^{2}\right) \times \pi_{0} \Delta^{\prime}(f)\right),
\end{gathered}
$$


see diagram (1.4), it follows that in order to describe them and the diagram (1.4) we need to characterize images of $\pi_{1} \mathcal{D}_{\mathrm{id}}\left(T^{2}\right)$ and $\pi_{0} \Delta^{\prime}(f)$ with respect to the maps $\xi_{1}^{-1}$ and $\xi_{2}^{-1}$ and take the corresponding quotient-groups. This will be done in Subsection 7.2 and Subsection 8.2.

\section{Proof of (1) of Theorem 3.2}

Due to our strategy, see Subsection 6.2, first we give the explicit description of the isomorphism $\xi_{1}: \mathcal{S}_{\mathbb{D}} \imath_{n, n m} \mathbb{Z}^{2} \rightarrow \pi_{1} \mathcal{O}_{f}(f)$.

7.1. Isomorphism from (1) of Theorem 6.1. Let $f$ be a Morse function on $T^{2}=\mathbb{R}^{2} / \mathbb{Z}^{2}$ such that its graph $\Gamma_{f}$ is a tree, $v$ be the special vertex of $\Gamma_{f}$, and $G_{v}^{\text {loc }}=\mathbb{Z}_{n} \times \mathbb{Z}_{n m}$ be the local stabilizer of $v$. Then the free action of $\mathbb{Z}_{n} \times \mathbb{Z}_{n m}$ on $T^{2}$ can be given by:

$$
\kappa_{(a, b)}(x, y)=\left(x+\frac{a}{n} \bmod 1, y+\frac{b}{n m} \bmod 1\right),
$$

for $(a, b) \in \mathbb{Z}_{n} \times \mathbb{Z}_{n m}$ and $(x, y) \in T^{2}$, and the quotient space $T^{2} / G_{v}^{l o c}$ is diffeomorphic to $T^{2}=\mathbb{R}^{2} / \mathbb{Z}^{2}$, so that the quotient map $q: T^{2} \rightarrow T^{2} / G_{v}^{\text {loc }}$ is given by the formula

$$
q(x, y)=(n x \bmod 1, n m y \bmod 1) .
$$

Let $y$ be a point in $T^{2}$, and $z=q(y) \in T^{2} / G_{v}^{l o c}$. Then we obtain the following commutative diagram with exact rows

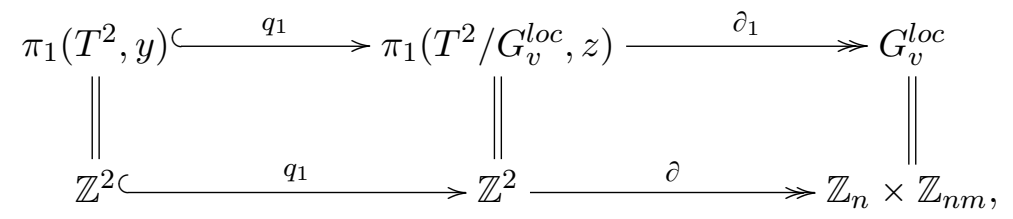

where the maps $q_{1}$ and $\partial$ are given as follows

$$
q_{1}(\lambda, \mu)=(n \lambda, n m \mu), \quad \partial(x, y)=(x \bmod n, y \bmod n m) .
$$

Let $\mathrm{L}, \mathrm{M}: T^{2} \times \mathbb{R} \rightarrow T^{2}$ be smooth flows on $T^{2}$ defined by

$$
\mathrm{L}(x, y, t)=\left(x+\frac{t}{n} \bmod 1, y, t\right), \quad \mathrm{M}(x, y, t)=\left(x, y+\frac{t}{n m} \bmod 1, t\right) .
$$

Then they commute each with other, and

$$
\mathrm{L}_{a}(x, y)=\kappa_{(a, 0)}(x, y), \quad \mathrm{M}_{b}(x, y)=\kappa_{(0, b)}(x, y),
$$

for $a, b \in \mathbb{R}$. Moreover, $\mathrm{L}_{n}=\mathrm{M}_{n m}=\mathrm{id}_{T^{2}}$, and the restrictions

$$
\mathrm{L}: T^{2} \times[0, n] \rightarrow T^{2}, \quad \mathrm{M}: T^{2} \times[0, n m] \rightarrow T^{2}
$$

can be regarded as loops in $\mathcal{D}_{\text {id }}\left(T^{2}\right)$ constituting also a basis $(1,0),(0,1)$ of $\pi_{1} \mathcal{D}_{\text {id }}\left(T^{2}\right) \cong \mathbb{Z}^{2}$. 
Then, [2], the isomorphism $\xi_{1}: \mathcal{S}_{\mathbb{D}} 2_{n, n m} \mathbb{Z}^{2} \rightarrow \pi_{1} \mathcal{O}_{f}(f)$, see (6.1), can be defined as follows. Let

$$
\left(\left\{h_{i j k}\right\},(a, b)\right) \in \mathcal{S}_{\mathbb{D}} l_{n, n m} \mathbb{Z}^{2},
$$

where $h_{i j k} \in \mathcal{S}^{\prime}\left(\left.f\right|_{D_{i 00}}, \partial D_{i 00}\right),(a, b) \in \mathbb{Z}^{2}, i=1, \ldots, r, j=0, \ldots, n-1$, $k=0, \ldots, n m-1$. For each triple $(i, j, k)$ fix any isotopy $h_{i j k}^{t}: D_{i 00} \rightarrow D_{i 00}$, $t \in[0,1]$ between $h_{i j k}^{0}=\operatorname{id}_{D_{i 00}}$ and $h_{i j k}^{1}=h_{i j k}$ relatively some neighborhood of $\partial D_{i 00}$. Then

$$
\xi_{1}\left(\left\{h_{i j k}\right\},(a, b)\right)=\left[\left\{f \circ h^{t}\right\}\right],
$$

where $h^{t}: T^{2} \rightarrow T^{2}, t \in[0,1]$, is given by the formula

$$
h^{t}(x)= \begin{cases}\left\{\mathrm{M}_{k+\frac{b t}{n m}} \circ \mathrm{L}_{j+\frac{a t}{n}} \circ h_{i j k}^{t} \circ \mathrm{L}_{j}^{-1} \circ \mathrm{M}_{k}^{-1}(x)\right\}_{i j k}, & x \in D_{i j k}, \\ \mathrm{M}_{\frac{b t}{n m}} \circ \mathrm{L}_{\frac{a t}{n}}(x), & x \in N,\end{cases}
$$

$D_{i j k}$ is a 2-disk defined in (ii) of Remark 4.3, and $N$ is a regular neighborhood of the critical level-set $V=p_{f}^{-1}(v)$ containing no other critical points.

7.2. Images of $\pi_{1} \mathcal{D}_{\mathrm{id}}\left(T^{2}\right)$ and $\pi_{0} \Delta^{\prime}(f)$ in $\mathcal{S}_{\mathbb{D}} 2_{n, n m} \mathbb{Z}^{2}$. The following lemma describes the images of the groups $\pi_{1} \mathcal{D}_{\text {id }}\left(T^{2}\right)$ and $\pi_{0} \Delta^{\prime}(f)$ with respect to the map $\xi_{1}^{-1}$.

Lemma 7.3. (1) Let $Z_{n, 0}=(\underbrace{e, \ldots, e}_{n m n}, n, 0)$ and $Z_{0, n m}=(\underbrace{e, \ldots, e}_{n m n}, 0, n m)$ be elements from $\mathcal{S}_{\mathbb{D}} l_{n, n m} \mathbb{Z}^{2}$, where $e$ is the unit of $\mathcal{S}_{\mathbb{D}}$. Then

$$
p_{1}(\mathrm{~L})=\xi_{1}\left(Z_{n, 0}\right), \quad p_{1}(\mathrm{M})=\xi_{1}\left(Z_{0, n m}\right) .
$$

(2) The isomorphism $\xi_{1}$ induces an isomorphism

$$
\left.\xi_{1}\right|_{\Delta_{\mathbb{D}}^{n m n}}:\left(\Delta_{\mathbb{D}}^{n m n}, 0,0\right) \rightarrow \iota_{1}\left(\pi_{0} \Delta^{\prime}(f)\right) .
$$

Proof. (1) Indeed, $\xi_{1}\left(Z_{n, 0}\right)$ is a loop $f \circ h^{t}$, where $h^{t}$ is given by (7.3) with $a=1$ and $b=0$. Then by (7.3) the isotopy $h$ has the form $h^{t}=\mathrm{L}_{t}$.

The case of $p_{1}(\mathrm{M})$ can be checked similarly and we leave this verification to the reader. So we have $p_{1}(\langle\mathrm{~L}\rangle)=\xi_{1}\left(\left\langle Z_{n, 0}\right\rangle\right)$ and $p_{1}(\langle\mathrm{M}\rangle)=\xi_{1}\left(\left\langle Z_{0, n m}\right\rangle\right)$.

(2) Let $\iota: \pi_{0} \Delta^{\prime}(f) \rightarrow \pi_{1} \mathcal{O}_{f}(f)$ be the restriction of $\iota_{1}$ onto $\pi_{0} \Delta^{\prime}(f)$. We need to show that $\xi_{1}$ isomorphically maps the subgroup $\left(\Delta_{\mathbb{D}}^{n m n}, 0,0\right)$ of $\mathcal{S}_{\mathbb{D}} \imath_{n, n m} \mathbb{Z}^{2}$ onto $\iota\left(\pi_{0} \Delta^{\prime}(f)\right)$. Obviously, an isomorphism $\xi_{1}$ induces a monomorphism $\left.\xi_{1}\right|_{\Delta_{\mathbb{D}}^{n m n}}: \Delta_{\mathbb{D}}^{n m n} \rightarrow \iota_{1}\left(\pi_{0} \Delta^{\prime}(f)\right)$. It remains to show that $\left.\xi_{1}\right|_{\Delta_{\mathbb{D}}^{n m n}}$ is an epimorphism.

Let $\tau$ be a Dehn twist supported on some 2-disk

$$
D \in\left\{D_{i, j, k}\right\}_{i=1, \ldots, r, j=0, \ldots, n-1, k=0, \ldots, n m-1},
$$


so it isotopy class $[\tau]$ is a free generator of a free abelian group $\pi_{0} \Delta^{\prime}(f)$.

Fix an isotopy $\tau^{t}: T^{2} \rightarrow T^{2}$ between $\tau^{1}=\tau$ and $\tau^{0}=\operatorname{id}_{T^{2}}$. The image $\iota([\tau])$ is given by the class of the loop $\left[f \circ \tau^{t}\right]$. It follows from (7.3) that there exist a diffeomorphism $\tilde{h}$ of $D$ and an isotopy $\tilde{h}^{t}: D \rightarrow D$ between $\tilde{h}^{1}=\tilde{h}$ and $\tilde{h}^{0}=\operatorname{id}_{D}$ such that $\xi_{1}(\{[\tilde{h}]\}, 0,0)=\left[f \circ \tau^{t}\right]$. Also we get from (7.3) that $\tilde{h}$ is conjugate to $\left.\tau\right|_{D}$, and so $\tilde{h}$ is a Dehn twist. Hence $\tilde{h}$ belongs to $\Delta_{\mathbb{D}}^{n m n}$. Thus $\left.\xi_{1}\right|_{\Delta_{\mathbb{D}}^{n m n}}$ is an isomorphism.

7.4. Final remarks to the proof of (1) of Theorem 3.2. Notice that by Diagram (1.4):

$$
\pi_{0} \mathcal{S}^{\prime}(f) \cong \pi_{1} \mathcal{O}_{f}(f) / \pi_{1} \mathcal{D}_{\mathrm{id}}\left(T^{2}\right), \quad G(f) \cong \pi_{0} \mathcal{S}^{\prime}(f) / \pi_{0} \Delta^{\prime}(f)
$$

The images of $\pi_{1} \mathcal{D}_{\text {id }}\left(T^{2}\right)$ and $\pi_{0} \Delta^{\prime}(f)$ in $\pi_{1} \mathcal{O}_{f}(f)$ are described in Subsection 7.2. Then one easily proves that $\xi_{1}$ induces the following isomorphisms

$$
\pi_{0} \mathcal{S}^{\prime}(f) \cong \mathcal{S}_{\mathbb{D}} \zeta\left(\mathbb{Z}_{n} \times \mathbb{Z}_{n m}\right), \quad G(f) \cong G_{\mathbb{D}} \zeta\left(\mathbb{Z}_{n} \times \mathbb{Z}_{n m}\right)
$$

as well as isomorphism of diagrams from (1) of Theorem 3.2.

\section{Proof of (2) of Theorem 3.2}

First we recall the definition of the isomorphism $\xi_{2}: \mathcal{S}_{Q} \imath_{n} \mathbb{Z} \rightarrow \pi_{1} \mathcal{O}_{f}(f)$.

8.1. Isomorphism from (2) of Theorem 6.1. Let $f$ be a Morse function from $\mathscr{F}_{1}$ with a cyclic index $n$ and circuit $\Theta$ in the graph $\Gamma_{f}$. As curves from $\mathcal{C}$ are "parallel", one can assume that the following conditions hold:

(a) $C_{i}=\frac{i}{n} \times S^{1} \subset \mathbb{R}^{2} / \mathbb{Z}^{2}=T^{2}$;

(b) there exists $\varepsilon \geqslant 0$ such that for all $t \in\left(\frac{i}{n}-\varepsilon, \frac{i}{n}+\varepsilon\right)$ the curve $t \times S^{1}$ is a regular connected component of some level set of $f$.

Let $\mathbb{L}, \mathbb{M}: T^{2} \times \mathbb{R} \rightarrow T^{2}$ be two flows defined by formulas:

$$
\mathbb{L}_{t}(x, y)=(x+t \bmod 1, y), \quad \mathbb{M}_{t}(x, y)=(x, y+t \bmod 1),
$$

$x \in C^{\prime}, y \in C_{0}$, and $t \in \mathbb{R}$.

Denote by $Q$ the cylinder bounded by $C_{0}$ and $C_{1}$. Then the isomorphism $\xi_{2}: \mathcal{S}_{Q} ?_{n} \mathbb{Z} \rightarrow \pi_{1} \mathcal{O}_{f}(f)$ can be defined as follows, [16, Section 8]. Let

$$
\left(h_{1}, \ldots, h_{n} ; a\right) \in \mathcal{S}_{Q} l_{n} \mathbb{Z},
$$

where $h_{i} \in \mathcal{S}_{Q}, i=0, \ldots, n$, and $a \in \mathbb{Z}$. Fix any isotopy $h_{i}^{t}$ between $h_{i}^{0}=\operatorname{id}_{Q}$ and $h_{i}^{1}=h_{i}$. Then

$$
\xi_{2}\left(h_{1}^{t}, \ldots, h_{n}^{t} ; a\right)=\left[f \circ h^{t}\right]
$$

where $h^{t}$ is defined by the formula

$$
h^{t}(x)=\left\{\mathbb{L}_{i+\frac{a t}{n}} \circ h_{i}^{t} \circ \mathbb{L}_{i}^{-1}(x)\right\}_{i=0, \ldots, n}, \quad x \in Q_{i} .
$$


8.2. Images of $\pi_{1} \mathcal{D}_{\text {id }}\left(T^{2}\right)$ and $\pi_{0} \Delta^{\prime}(f)$ in $\mathcal{S}_{Q} \imath_{n} \mathbb{Z}$. The following lemma easily follows from the definition of the isomorphism $\xi_{2}$ similarly to (1) of Lemma 7.3.

Lemma 8.3. Let $Z=(\underbrace{e^{\prime}, \ldots, e^{\prime}}_{n}, n)$ be the element from $S_{Q} \imath_{n} \mathbb{Z}$, where $e^{\prime}$ is the unit of $\mathcal{S}_{Q}$. Then $p_{1}(\mathbb{L})=\xi_{2}(Z)$.

The image of another generator $\mathbb{M}$ in $\pi_{1} \mathcal{O}_{f}(f)$ is "invisible" in our description of the group $\pi_{1} \mathcal{O}_{f}(f)$ via $\mathcal{S}_{Q} \imath_{n} \mathbb{Z}$, however in [16, Theorem 6] we showed that $p_{1}(\mathbb{M})$ in $\pi_{1} \mathcal{O}_{f}(f)$ is given by $[f \circ \theta]$, where $\theta=\theta_{0} \circ \ldots \circ \theta_{n-1}$ is the generator of $H=\langle\theta\rangle$, and $\theta_{i}$ is a slide along $C_{i}$, see Section 5.3. Then by Theorem 5.5 groups $\pi_{0} \Delta^{\prime}(f) \times\langle\mathbb{M}\rangle$ and $\pi_{0} \Delta^{\prime}(f, \mathcal{C})$ are isomorphic as subgroups of $\pi_{1} \mathcal{O}_{f}(f)$. So $\pi_{0} \Delta^{\prime}(f) \cong \pi_{0} \Delta^{\prime}(f, \mathcal{C}) / H$.

Lemma 8.4. The isomorphism $\xi_{2}$ induces an isomorphism

$$
\left.\xi_{2}\right|_{\Delta_{Q}^{n}}:\left(\Delta_{Q}^{n}, 0\right) \rightarrow \iota_{1}\left(\pi_{0} \Delta^{\prime}(f, \mathcal{C})\right),
$$

where $\Delta_{Q}=\pi_{0} \Delta^{\prime}\left(\left.f\right|_{Q}, \partial Q\right)$. So $\pi_{0} \Delta^{\prime}(f)$ is isomorphic to $\Delta_{Q}^{n} / H$.

Proof. Obviously, the isomorphism $\xi_{2}$ induces a monomorphism $\left.\xi_{2}\right|_{\Delta_{Q}^{n}}$. Then $\tau \in \Delta^{\prime}(f, \mathcal{C})$ be a Dehn twist supported in $Q \in\left\{Q_{i}\right\}_{i=0, \ldots, n-1}$, such that its isotopy class $[\tau]$ is one of free generators of the free abelian group $\pi_{0} \Delta^{\prime}(f, \mathcal{C})$. Fix an isotopy $\tau^{t}: T^{2} \rightarrow T^{2}$ such that $\tau^{1}=\tau$ and $\tau^{0}=$ id. Then $\iota([\tau])=\left[f \circ \tau^{t}\right]$. From (8.1) there exists a diffeomorphism $\tilde{h}: Q \rightarrow Q$ and an isotopy $\tilde{h}^{t}: Q \rightarrow Q$ between $\tilde{h}^{1}=\tilde{h}$ and $\tilde{h}^{0}=\operatorname{id}_{Q}$ such that $\xi_{2}([\tilde{h}], 0)=\left[f \circ \tau^{t}\right]$. By $(8.2) \tilde{h}$ is conjugate to $\tau_{Q}$, then $\tilde{h}$ is also a Dehn twist. Hence $\tilde{h}$ belongs to $\Delta_{Q}^{n}$. So $\left.\xi_{2}\right|_{\Delta_{Q}^{n}}$ is an isomorphism, and hence $\pi_{0} \Delta^{\prime}(f)$ is isomorphic to $\Delta_{Q}^{n} / H$.

The following corollary directly follows from Lemma 5.2 and describes $\pi_{1} \mathcal{O}_{f}(f)$ for functions from $\mathscr{F}_{1}$ via embedded 2-disks and the image of the generator $\mathbb{M}$ in $\pi_{1} \mathcal{O}_{f}(f)$.

Corollary 8.5. (1) There exist a cylinder $Q$ and a set of mutually disjoint 2-disks $\mathbb{Y}=\left\{Y_{i j}\right\}_{i=0, \ldots, k}^{j=0, \ldots, c_{i}} \subset Q$ for some $k, c_{i} \in \mathbb{N}$, and $m_{i} \in \mathbb{N}$, $i=1, \ldots, k$ such that the following groups are isomorphic

$$
\pi_{1} \mathcal{O}_{f}(f) \stackrel{\xi_{2}^{-1}}{=} \mathcal{S}_{Q} \imath_{n} \mathbb{Z} \stackrel{\zeta^{\prime}}{\cong} \mathcal{S}_{\mathbb{Y}} \imath_{n} \mathbb{Z}
$$

where $\zeta^{\prime}=\underbrace{\zeta \times \ldots \times \zeta \times \mathrm{id}_{\mathbb{Z}}}$. 
(2) The group $H$ is normal in $\Delta_{\mathbb{Y}}^{n}$ is generated by

$$
(\underbrace{\left(E_{1}, m_{1}, E_{2}, m_{2}, \ldots, E_{k}, m_{k}\right), \ldots,\left(E_{1}, m_{1}, E_{2}, m_{2}, \ldots, E_{k}, m_{k}\right)}_{n},
$$

where $E_{i}$ is the unit of the group $\left(\prod_{j=1}^{c_{i}} \Delta_{Y_{i j}}\right)^{m_{i}}$, and the image of $H$ in $\mathcal{S}_{\mathbb{Y}} l_{n} \mathbb{Z}$ is generated by

$$
\left.(\underbrace{\left(E_{1}, m_{1}, E_{2}, m_{2}, \ldots, E_{k}, m_{k}\right), \ldots,\left(E_{1}, m_{1}, E_{2}, m_{2}, \ldots, E_{k}, m_{k}\right.}_{n}), 0\right),
$$

8.6. Final remarks for the proof of (2) of Theorem 3.2. Similarly to the proof of (1) of Theorem 3.2, groups $\pi_{0} \mathcal{S}^{\prime}(f)$ and $G(f)$ are the corresponding quotient-groups $\pi_{1} \mathcal{O}_{f}(f) / \pi_{1} \mathcal{D}_{\text {id }}\left(T^{2}\right)$ and $G(f)=\pi_{0} \mathcal{S}^{\prime}(f) / \pi_{0} \Delta^{\prime}(f)$, see diagram (1.4). The images of $\pi_{1} \mathcal{D}_{\text {id }}\left(T^{2}\right)$ and $\pi_{0} \Delta^{\prime}(f)$ in $\pi_{1} \mathcal{O}_{f}(f)$ are known, see Subsection 8.2. So it is easy to prove that an isomorphism $\zeta^{\prime} \circ \xi_{2}$ induces the following isomorphisms

$$
\pi_{0} \mathcal{S}^{\prime}(f) \cong\left(\mathcal{S}_{\mathbb{Y}} \succ \mathbb{Z}_{n}\right) / H, \quad G(f) \cong G_{\mathbb{Y}} \succ \mathbb{Z}_{n}
$$

and so it induces an isomorphism of diagrams from (2) of Theorem 3.2.

\section{REFERENCES}

[1] C. J. Earle, J. Eells. A fibre bundle description of teichmüller theory. J. Differential Geometry, 3:19-43, 1969.

[2] B. Feshhenko. Deformations of smooth functions on 2-torus, whose kr-graph is a tree. Proceedings of Institute of Mathematics of NAS of Ukraine, 12(6):22-40, 2015.

[3] André Gramain. Le type d'homotopie du groupe des difféomorphismes d'une surface compacte. Ann. Sci. École Norm. Sup. (4), 6:53-66, 1973.

[4] Kazuichi Ikegami, Osamu Saeki. Cobordism group of Morse functions on surfaces. $J$. Math. Soc. Japan, 55(4):1081-1094, 2003, doi: 10.2969/jms j/1191418765.

[5] Boldizsár Kalmár. Cobordism group of Morse functions on unoriented surfaces. Kyushu J. Math., 59(2):351-363, 2005, doi: 10.2206/kyushujm.59.351.

[6] E. A. Kudryavtseva. Realization of smooth functions on surfaces as height functions. Mat. Sb., 190(3):29-88, 1999, doi: 10.1070/SM1999v190n03ABEH000392.

[7] E. A. Kudryavtseva. The topology of spaces of Morse functions on surfaces. Math. Notes, 92(1-2):219-236, 2012, doi: 10.1134/S0001434612070243. Translation of Mat. Zametki 92 (2012), no. 2, 241-261.

[8] E. A. Kudryavtseva. On the homotopy type of spaces of Morse functions on surfaces. Mat. Sb., 204(1):79-118, 2013, doi: 10.1070/SM2013v204n01ABEH004292.

[9] S. Maksymenko, B. Feshchenko. Homotopy properties of spaces of smooth functions on2-torus. Ukrainian Mathematical Journal, 66(9):1205-1212, 2014.

[10] Sergiy Maksymenko. Homotopy types of stabilizers and orbits of Morse functions on surfaces. Ann. Global Anal. Geom., 29(3):241-285, 2006.

[11] Sergiy Maksymenko. Functions on surfaces and incompressible subsurfaces. Methods Funct. Anal. Topology, 16(2):167-182, 2010.

[12] Sergiy Maksymenko. Functions with isolated singularities on surfaces. Geometry and topology of functions on manifolds. Pr. Inst. Mat. Nats. Akad. Nauk Ukr. Mat. Zastos., $7(4): 7-66,2010$. 
[13] Sergiy Maksymenko. Local inverses of shift maps along orbits of flows. Osaka Journal of Mathematics, 48(2):415-455, 2011.

[14] Sergiy Maksymenko. Homotopy types of right stabilizers and orbits of smooth functions functions on surfaces. Ukrainian Math. Journal, 64(9):1186-1203, 2012 (in Russian).

[15] Sergiy Maksymenko. Deformations of functions on surfaces by isotopic to the identity diffeomorphisms. page arXiv:math/1311.3347, 2014.

[16] Sergiy Maksymenko, Bohdan Feshchenko. Functions on 2-torus whose kronrod-reeb graph contains a cycle. Methods of Functional Analysis and Topology, 21(1):22-40, 2015 .

[17] Sergiy Maksymenko, Bohdan Feshchenko. Orbits of smooth functions on 2-torus and their homotopy types. Matematychni Studii, 44(1):67-83, 2015.

[18] J. D. P. Meldrum. Wreath products of groups and semigroups, volume 74 of Pitman Monographs and Surveys in Pure and Applied Mathematics. Longman, Harlow, 1995.

[19] Francis Sergeraert. Un théorème de fonctions implicites sur certains espaces de Fréchet et quelques applications. Ann. Sci. École Norm. Sup. (4), 5:599-660, 1972.

[20] V. V. Sharko. Functions on surfaces. I. In Some problems in contemporary mathematics (Russian), volume 25 of Pr. Inst. Mat. Nats. Akad. Nauk Ukr. Mat. Zastos., pages $408-$ 434. Natsīonal. Akad. Nauk Ukraïni, Inst. Mat., Kiev, 1998.

\section{Bohdan Feshchenko}

Topology laboratory, Department of algebra and topology, Institute of Mathematics of National Academy of Science of Ukraine, Tereshchenkivska, 3, Kyiv, 01024, UKRAINE

Email: fb@imath.kiev.ua 